\title{
On Semi-Invariant Submanifoldsof A Nearly Hyperbolic Kenmotsu Manifold With Semi-Symmetric Semi-Metric Connection
}

\author{
Mobin Ahmad ${ }^{1}$, Shadab Ahmad Khan ${ }^{2}$ and Toukeer Khan ${ }^{3}$ \\ ${ }^{I}$ Department of Mathematics, Faculty of Science, Jazan University, Jazan-2069, Saudi Arabia. \\ 2,3 Department of Mathematics, Integral University, Kursi Road, Lucknow-226026, India.
}

\begin{abstract}
Weconsiders a nearly hyperbolic Kenmotsu manifold admitting a semi-symmetric semi-metric connection and study semi-invariant submanifolds of a nearly hyperbolic Kenmotsu manifold with semisymmetric semi-metric connection. We also find the integrability conditions of some distributions on nearly hyperbolic Kenmotsu manifold and study parallel distributions (horizontal \& vertical distributions) on nearly hyperbolic Kenmotsu manifold.
\end{abstract}

Key Words and Phrases:Semi-invariant submanifolds, Nearly hyperbolic Kenmotsu manifold, Parallel distribution, Integrability condition \& Semi-symmetric semi-metric connection.

2000 AMS Mathematics Subject Classification:53D05, 53D25, 53D12.

\section{Introduction}

The study of CR-submanifolds of Kaehler manifold as generalization of invariant and anti-invariant submanifolds was initiated by A. Bejancu in [8]. A semi-invariant submanifold is the extension of a CRsubmanifold of a Kaehler manifold to submanifolds of almost contact manifolds. The study of semi- invariant submanifolds of Sasakian manifolds was initiated by Bejancu-Papaghuic in [10]. The same concept was studied under the name contact CR-submanifold by Yano-Kon in [19] and K. Matsumoto in [16]. The study of semi-invariant submanifolds in almost contact manifold was enriched by several geometers (see, [2], [4], [5], [6], [7], [8], [12], [13], [17]). On the otherhand, almost hyperbolic $(f, \xi, \eta, g)$, structure was defined and studied by Upadhyay and Dube in [18].Joshi and Dube studied semi-invariant submanifolds of an almost $r$ contact hyperbolic metric manifold in [15].Motivated by the studies in ([2], [3], [4], [5], [6], [7], [8], [9]), in this paper, we study semi-invariant submanifolds of a nearly hyperbolic Kenmotsu manifold admitting a semisymmetric semi-metric connection.

Let $\nabla$ be a linear connection in an n-dimensional differentiable manifold $\bar{M}$. The torsion tensor $T$ and curvature tensor $R$ of $\nabla$ given respectively by

$$
\begin{gathered}
T(X, Y)=\nabla_{X} Y-\nabla_{Y} X-[X, Y], \\
R(X, Y) Z=\nabla_{X} \nabla_{Y} Z-\nabla_{Y} \nabla_{X} Z-\nabla_{[X, Y]} Z .
\end{gathered}
$$

The connection $\nabla$ is symmetric if its torsion tensor $T$ vanishes, otherwise it is non-symmetric. The connection $\nabla$ is metric connection if there is a Riemannian metric $\mathrm{g}$ in $\bar{M}$ such that $\nabla g=0$, otherwise it is nonmetric. It is well known that a linear connection is symmetric and metric if and only if it is the Levi-Civita connection. A. Friedmann and J. A. Schouten introduced the idea of a semi-symmetric connection. A linear connection is said to be a semi-symmetric connection if its torsion tensor $\mathrm{T}$ is of the form:

$$
T(X, Y)=\eta(X) Y-\eta(Y) X .
$$

Many geometers (see, [2], [20], [21]) have studied certain properties of semi-symmetric semi-metric connection. This paper is organized as follows. In section 2, we give a brief description of nearly hyperbolic Kenmotsu manifold admitting a semi-symmetric semi-metric connection. In section 3, we study some properties of semi-invariant submanifolds of a nearly hyperbolic Kenmotsu manifold with a semi-symmetric semi-metric connection. In section 4, we discuss the integrability conditions of some distributions on nearly hyperbolic Kenmotsu manifold with a semi-symmetric semi-metric connection. In section 5, we obtain parallel horizontal distribution on nearly hyperbolic Kenmotsu manifold with a semi-symmetric semi-metric connection.

\section{Preliminaries}

Let $\bar{M}$ be an $n$-dimensional almost hyperbolic contact metric manifold with the almost hyperbolic contact metric structure $(\varnothing, \xi, \eta, g)$, where a tensor $\emptyset$ of type $(1,1)$ a vector field $\xi$, called structure vector field and $\eta$, the dual 1-form of $\xi$ and the associated Riemannian metric $g$ satisfying the following

$$
\begin{aligned}
& \emptyset^{2} X=X+\eta(X) \xi, \quad g(X, \xi)=\eta(X), \\
& \eta(\xi)=-1, \quad \emptyset(\xi)=0, \quad \eta \varnothing \emptyset=0, \\
& g(\varnothing X, \varnothing Y)=-g(X, Y)-\eta(X) \eta(Y)
\end{aligned}
$$


for any $X, Y$ tangent to $\bar{M}[19]$. In this case

$$
g(\varnothing X, Y)=-g(\varnothing Y, X) .
$$

An almost hyperbolic contact metric structure $(\varnothing, \xi, \eta, g)$ on $\bar{M}$ is called hyperbolic Kenmotsu manifold [7] if and only if

for all $X, Y$ tangent to $\bar{M}$.

$$
\left(\nabla_{X} \emptyset\right) Y=g(\varnothing X, Y) \xi-\eta(Y) \emptyset X
$$

On a hyperbolic Kenmotsumanifold $\bar{M}$, we have

$$
\nabla_{X} \xi=X+\eta(X) \xi
$$

for a Riemannian metric $g$ and Riemannian connection $\nabla$.

Further, an almost hyperbolic contact metric manifold $\bar{M}$ on $(\varnothing, \xi, \eta, g)$ is called a nearly hyperbolic Kenmotsu manifold [7], if

where $\nabla$ is Riemannian connection $\bar{M}$.

$$
\left(\nabla_{X} \emptyset\right) Y+\left(\nabla_{Y} \emptyset\right) X=-\eta(X) \emptyset Y-\eta(Y) \emptyset X,
$$

Now, we define a semi-symmetric semi-metric connection

$$
\bar{\nabla}_{X} Y=\nabla_{X} Y-\eta(X) Y+g(X, Y) \xi
$$

such that $\left(\bar{\nabla}_{X} g\right)(Y, Z)=2 \eta(X) g(Y, Z)-\eta(Y) g(X, Z)-\eta(Z) g(X, Y)$.

From (2.7)\& (2.8), we obtain

$$
\begin{gathered}
\left(\bar{\nabla}_{X} \emptyset\right) Y+\left(\bar{\nabla}_{Y} \emptyset\right) X=-\eta(X) \emptyset Y-\eta(Y) \emptyset X \\
\bar{\nabla}_{X} \xi=X+\eta(X) \xi .
\end{gathered}
$$

An almost hyperbolic contact metric manifold with almost hyperbolic contact structure $(\varnothing, \xi, \eta, g)$ is called nearly hyperbolic Kenmotsu manifold with semi-symmetric semi-metric connection if it satisfy (2.9) and (2.10).

For semi-symmetric semi-metric connection, the Nijenhuis tensor is expressed as

$$
N(X, Y)=\left(\bar{\nabla}_{\varnothing X} \emptyset\right) Y-\left(\bar{\nabla}_{\varnothing Y} \emptyset\right) X-\emptyset\left(\bar{\nabla}_{X} \emptyset\right) Y+\emptyset\left(\bar{\nabla}_{Y} \emptyset\right) X .
$$

Now from (2.9), we get

$$
\left(\bar{\nabla}_{\emptyset X} \emptyset\right) Y=-\eta(Y) X-\eta(X) \eta(Y) \xi-\left(\bar{\nabla}_{Y} \varnothing\right) \emptyset X .
$$

Differentiating (2.1) conveniently along the vector Yand using (2.10), we have

From (2.12) and (2.13), we have

$$
\left(\bar{\nabla}_{Y} \emptyset\right) \emptyset X=\left(\bar{\nabla}_{Y} \eta\right)(X) \xi+\eta(X) Y+\eta(X) \eta(Y) \xi-\emptyset\left(\bar{\nabla}_{Y} \emptyset\right) X .
$$

$$
\left(\bar{\nabla}_{\varnothing X} \emptyset\right) Y=-\eta(X) Y-\eta(Y) X-2 \eta(X) \eta(Y) \xi-\left(\bar{\nabla}_{Y} \eta\right)(X) \xi+\emptyset\left(\bar{\nabla}_{Y} \emptyset\right) X .
$$

Interchanging $X$ and, $Y$ we have

$$
\left(\bar{\nabla}_{\varnothing Y} \emptyset\right) X=-\eta(Y) X-\eta(X) Y-2 \eta(X) \eta(Y) \xi-\left(\bar{\nabla}_{X} \eta\right)(Y) \xi+\emptyset\left(\bar{\nabla}_{X} \emptyset\right) Y .
$$

Using equation (2.14), (2.15) in (2.11), we obtain

Using equation (2.9), we have

$$
N(X, Y)=2 d \eta(X, Y) \xi+2 \varnothing\left(\bar{\nabla}_{Y} \emptyset\right) X-2 \varnothing\left(\bar{\nabla}_{X} \emptyset\right) Y
$$

As we know that $\left(\bar{\nabla}_{Y} \emptyset\right) X=\bar{\nabla}_{Y} \emptyset X-\emptyset\left(\bar{\nabla}_{Y} X\right)$, usingGuass formula, we have

$$
N(X, Y)=2 g(\varnothing X, Y) \xi+4 \varnothing\left(\bar{\nabla}_{Y} \emptyset\right) X+2 \eta(X) Y+2 \eta(Y) X+4 \eta(X) \eta(Y) \xi
$$

Using this equation in (2.17), we have

$$
\emptyset\left(\bar{\nabla}_{Y} \emptyset\right) X=\emptyset\left(\nabla_{Y} \emptyset X\right)+\emptyset h(Y, \varnothing X)-\nabla_{Y} X-\eta\left(\nabla_{Y} X\right) \xi-h(Y, X)
$$

$$
\begin{gathered}
N(X, Y)=2 \eta(X) Y+2 \eta(Y) X+4 \eta(X) \eta(Y) \xi+4 \emptyset\left(\nabla_{Y} \varnothing X\right)+4 \emptyset h(Y, \emptyset X) \\
-4\left(\nabla_{Y} X\right)-4 \eta\left(\nabla_{Y} X\right) \xi-4 h(Y, X)+2 g(\varnothing X, Y) \xi
\end{gathered}
$$

\section{Semi-invariant Submanifold}

Let $M$ be submanifold immersed in $\bar{M}$, we assume that the vector $\xi$ is tangent to $M$, denoted by $\{\xi\}$ the 1-dimentional distribution spanned by $\xi$ on $M$, then $M$ is called a semi-invariant submanifold [9] of $M$ if there exist two differentiable distribution $D \& D^{\perp}$ on $M$ satisfying

(i) $T M=D \oplus D^{\perp} \oplus \xi$, where $D, D^{\perp}$ and $\xi$ are mutually orthogonal to each other,

(ii) the distribution $D$ is invariant under $\emptyset$ that is $\emptyset D_{X}=D_{X}$ for each $X \in M$,

(iii) the distribution $D^{\perp}$ is anti-invariant under $\varnothing$, that is $\emptyset D^{\perp}{ }_{X} \subset T^{\perp} M$ for each $X \in M$, where $T M$ and $T^{\perp} M$ be the Lie algebra of vector fields tangential and normal to $M$ respectively.Let $g$ be the Riemannian metricand $\nabla$ be induced Levi-Civita connection on $M$, then the Guass and Weingarten formulas are given by

$$
\begin{array}{r}
\bar{\nabla}_{X} Y=\nabla_{X} Y+h(X, Y), \\
\bar{\nabla}_{X} N=-A_{N} X+\nabla_{X}^{\perp} N-\eta(X) N
\end{array}
$$

for any $X, Y \in T M$ and $N \in T^{\perp} M$, where $\nabla^{\perp}$ is a connection on the normal bundle $T^{\perp} M$, $h$ is the second fundamental form and $A_{N}$ is the Weingarten map associated with $N$ as

Any vector $X$ tangent to $M$ is given as

$$
g\left(A_{N} X, Y\right)=g(h(X, Y), N) .
$$

$$
X=P X+Q X+\eta(X) \xi,
$$


where $P X \in D$ and $Q X \in D^{\perp}$.

Similarly, for $N$ normal to $M$, we have

$$
\emptyset N=B N+C N,
$$

where $B N($ resp. $C N)$ is the tangential component (resp. normal component) of $\emptyset N$.

Theorem 3.1.The connection induced on a semi-invariant submanifold of a nearly hyperbolic Kenmotsu manifold with semi-symmetric semi-metric connection is also semi-symmetric semi-metric.

Proof:Let $\nabla$ be induced connection with respect to the normal $N$ on semi-invariant submanifold of nearly hyperbolic Kenmotsu manifold with semi-symmetric semi-metric connection $\bar{\nabla}$, then

$$
\bar{\nabla}_{X} Y=\nabla_{X} Y+m(X, Y)
$$

where $\mathrm{m}$ is the tensor filed of type $(0,2)$ on semi-invariant submanifold $M$. If $\nabla^{*}$ be the induced connection on semi-invariant submanifold from Riemannian connection $\overline{\bar{\nabla}}$, then

$$
\overline{\bar{\nabla}}_{X} Y=\nabla_{X}^{*} Y+h(X, Y) \text {, }
$$

whereh is second fundamental tensor \& we know that semi-symmetric semi-metric connection on nearly hyperbolic Kenmotsu manifold

Using (3.6), (3.7) in (3.8), we have

$$
\bar{\nabla}_{X} Y=\overline{\bar{\nabla}}_{X} Y-\eta(X) Y+g(X, Y) \xi .
$$

Comparing tangent and normal parts, we have

$$
\nabla_{X} Y+m(X, Y)=\nabla_{X}^{*} Y+h(X, Y)-\eta(X) Y+g(X, Y) \xi .
$$

$$
\begin{gathered}
\nabla_{X} Y=\nabla_{X}^{*} Y-\eta(X) Y+g(X, Y) \xi \\
m(X, Y)=h(X, Y)
\end{gathered}
$$

Thus $\nabla$ is also semi-symmetric semi-metric connection.

Lemma 3.2. Let $M$ be a semi-invariant submanifold of a nearly hyperbolic Kenmotsu manifold $\bar{M}$ with semisymmetric semi-metric connection, then

for each $X, Y \in D$.

$$
2\left(\bar{\nabla}_{X} \emptyset\right) Y=\nabla_{X} \varnothing Y-\nabla_{Y} \varnothing X+h(X, \varnothing Y)-h(Y, \varnothing X)-\emptyset[X, Y]
$$

Proof.From Gauss formula (3.1), we have

By covariant differentiation, we have

$$
\bar{\nabla}_{X} \emptyset Y-\bar{\nabla}_{Y} \varnothing X=\nabla_{X} \varnothing Y-\nabla_{Y} \emptyset X+h(X, \varnothing Y)-h(Y, \varnothing X) \text {. }
$$

From (3.10) and (3.11), we have

$$
\bar{\nabla}_{X} \varnothing Y-\bar{\nabla}_{Y} \varnothing X=\left(\bar{\nabla}_{X} \varnothing\right) Y-\left(\bar{\nabla}_{Y} \emptyset\right) X+\emptyset[X, Y] .
$$

$\left(\bar{\nabla}_{X} \varnothing\right) Y-\left(\bar{\nabla}_{Y} \emptyset\right) X=\nabla_{X} \varnothing Y-\nabla_{Y} \emptyset X+h(X, \varnothing Y)-h(Y, \varnothing X)-\emptyset[X, Y]$.

Adding (2.9) and (3.12), we obtain

for each $X, Y \in D$.

$$
2\left(\bar{\nabla}_{X} \varnothing\right) Y=\nabla_{X} \varnothing Y-\nabla_{Y} \varnothing X+h(X, \varnothing Y)-h(Y, \varnothing X)-\emptyset[X, Y]
$$

Lemma 3.3. Let $M$ be a semi-invariant submanifold of a nearly hyperbolic Kenmotsu manifold $\bar{M}$ with semisymmetric semi-metric connection, then

for all $X \in D$ and $Y \in D^{\perp}$.

$$
2\left(\bar{\nabla}_{X} \emptyset\right) Y=-A_{\varnothing Y} X+\nabla_{X}^{\frac{1}{X}} \emptyset Y-\nabla_{Y} \emptyset X-h(Y, \varnothing X)-\emptyset[X, Y]
$$

Proof.By Gauss formula (3.1), we have

Also, by Weingarten formula (3.2), we have

$$
\bar{\nabla}_{Y} \varnothing X=\nabla_{Y} \varnothing X+h(Y, \varnothing X)
$$

Subtracting abovetwo equations, we have

$$
\bar{\nabla}_{X} \emptyset Y=-A_{\varnothing Y} X+\nabla_{X}^{\frac{1}{X}} \emptyset Y-\eta(X) \emptyset Y \text {. }
$$

$$
\bar{\nabla}_{X} \varnothing Y-\bar{\nabla}_{Y} \varnothing X=-A_{\varnothing Y} X+\nabla_{X}^{\frac{1}{X}} \emptyset Y-\eta(X) \emptyset Y-\nabla_{Y} \emptyset X-h(Y, \emptyset X) .
$$

Comparing equation (3.11) and (3.13), we have

$$
\left(\bar{\nabla}_{X} \varnothing\right) Y-\left(\bar{\nabla}_{Y} \emptyset\right) X=-A_{\varnothing Y} X+\nabla_{X}^{\frac{1}{X}} \emptyset Y-\eta(X) \emptyset Y-\nabla_{Y} \emptyset X-h(Y, \varnothing X)-\emptyset[X, Y] .
$$

Adding equation (2.9) in above, we get

for all $X \in D$ and $Y \in D^{\perp}$.

$$
2\left(\bar{\nabla}_{X} \emptyset\right) Y=-A_{\varnothing Y} X+\nabla_{X}^{\frac{1}{X}} \varnothing Y-\nabla_{Y} \varnothing X-h(Y, \varnothing X)-\emptyset[X, Y] .
$$

Lemma 3.4.Let $M$ be a semi-invariant submanifold of a nearly hyperbolic Kenmotsu manifold $\bar{M}$ with semisymmetric semi-metric connection, then

for all $X, Y \in D^{\perp}$.

$$
2\left(\bar{\nabla}_{X} \emptyset\right) Y=A_{\emptyset X} Y-A_{\varnothing Y} X+\nabla_{X}^{1} \emptyset Y-\nabla_{Y}^{\frac{1}{Y}} \emptyset X-\emptyset[X, Y]
$$

Proof.Using Weingarten formula (3.2), we have 
Interchanging and $Y$, we have

$$
\bar{\nabla}_{X} \emptyset Y=-A_{\varnothing Y} X+\nabla_{X}^{\frac{1}{X}} \emptyset X-\eta(X) \emptyset Y .
$$

$$
\bar{\nabla}_{Y} \varnothing X=-A_{\varnothing X} Y+\nabla_{Y}^{\perp} \emptyset X-\eta(Y) \emptyset X .
$$

Subtracting equation (3.16) from (3.15), we have

$$
\bar{\nabla}_{X} \emptyset Y-\bar{\nabla}_{Y} \emptyset X=A_{\varnothing X} Y-A_{\emptyset Y} X+\nabla_{X}^{\frac{1}{X}} \emptyset Y-\nabla_{Y}^{\frac{1}{Y}} \emptyset X+\eta(Y) \emptyset X-\eta(X) \emptyset Y .
$$

Using equation (3.11) in (3.17), we have

Adding (2.9) in above equation, we have

$$
\begin{aligned}
&\left(\bar{\nabla}_{X} \emptyset\right) Y-\left(\bar{\nabla}_{Y} \emptyset\right) X=A_{\varnothing X} Y-A_{\emptyset Y} X+\nabla_{X}^{\frac{1}{X}} \emptyset Y-\nabla_{Y}^{1} \emptyset X \\
&+\eta(Y) \emptyset X-\eta(X) \emptyset Y-\emptyset[X, Y]
\end{aligned}
$$

for all $X, Y \in D^{\perp}$.

$$
2\left(\bar{\nabla}_{X} \emptyset\right) Y=A_{\varnothing X} Y-A_{\emptyset Y} X+\nabla_{X}^{\frac{1}{X}} \emptyset Y-\nabla_{Y}^{\perp} \emptyset X-\emptyset[X, Y]
$$

Lemma 3.5. Let $M$ be a semi-invariant submanifold of a nearly hyperbolic Kenmotsu manifold $\bar{M}$ with semisymmetric semi-metric connection, then

for all $X, Y \in T M$.

$$
\begin{aligned}
& P\left(\nabla_{X} \emptyset P Y\right)+P\left(\nabla_{Y} \emptyset P X\right)-P A_{\varnothing Q Y} X-P A_{\varnothing Q X} Y=-\eta(Y) \emptyset P X \\
& -\eta(X) \emptyset P Y+\emptyset P\left(\nabla_{X} Y\right)+\emptyset P\left(\nabla_{Y} X\right) \\
& Q\left(\nabla_{X} \emptyset P Y\right)+Q\left(\nabla_{Y} \emptyset P X\right)-Q A_{\emptyset Q Y} X-Q A_{\emptyset Q X} Y=2 B h(X, Y), \\
& h(Y, \emptyset P X)+h(X, \emptyset P Y)+\nabla_{X}^{\frac{1}{X}} \emptyset Q Y+\nabla_{Y}^{\frac{1}{Y}} \emptyset Q X=\emptyset Q\left(\nabla_{X} Y\right) \\
& +\emptyset Q\left(\nabla_{Y} X\right)+2 C h(X, Y) \text {, } \\
& \eta\left(\nabla_{X} \emptyset P Y\right)+\eta\left(\nabla_{Y} \emptyset P X\right)-\eta\left(A_{\varnothing Q Y} X\right)-\eta\left(A_{\emptyset Q X} Y\right)=0
\end{aligned}
$$

Proof.Differentiating covariantlyequation (3.4) and using equation (3.1) and (3.2), we have

Interchanging $X$ and $Y$, we have

$$
\begin{gathered}
\left(\bar{\nabla}_{X} \emptyset\right) Y+\emptyset\left(\nabla_{X} Y\right)+\emptyset h(X, Y)=\nabla_{X} \emptyset P Y+h(X, \emptyset P Y) \\
-A_{\varnothing Q Y} X+\nabla_{X}^{\perp} \emptyset Q Y-\eta(X) \emptyset Q Y .
\end{gathered}
$$

Adding above two equations, we get

$$
\begin{gathered}
\left(\bar{\nabla}_{Y} \emptyset\right) X+\emptyset\left(\nabla_{Y} X\right)+\emptyset h(Y, X)=\nabla_{Y} \emptyset P X+h(Y, \emptyset P X) \\
-A_{\emptyset Q X} Y+\nabla_{Y}^{\perp} \emptyset Q X-\eta(Y) \emptyset Q X .
\end{gathered}
$$

$$
\begin{gathered}
\left(\bar{\nabla}_{X} \emptyset\right) Y+\left(\bar{\nabla}_{Y} \emptyset\right) X+\emptyset\left(\nabla_{X} Y\right)+\emptyset\left(\nabla_{Y} X\right)+2 \emptyset h(X, Y)=\nabla_{X} \emptyset P Y+\nabla_{Y} \emptyset P X \\
+h(X, \emptyset P Y)+h(Y, \emptyset P X)-A_{\emptyset Q Y} X-A_{\emptyset Q X} Y+\nabla_{X}^{1} \emptyset Q Y \\
+\nabla_{Y}^{\perp} \emptyset Q X-\eta(X) \emptyset Q Y-\eta(Y) \emptyset Q X .
\end{gathered}
$$

Using equation (2.9) in above, we have

$$
\begin{gathered}
-\eta(X) \emptyset Y-\eta(Y) \emptyset X+\emptyset\left(\nabla_{X} Y\right)+\emptyset\left(\nabla_{Y} X\right)+2 \emptyset h(X, Y)=\nabla_{X} \emptyset P Y+\nabla_{Y} \emptyset P X \\
+h(X, \emptyset P Y)+h(Y, \emptyset P X)-A_{\varnothing Q Y} X-A_{\emptyset Q X} Y+\nabla_{X}^{\frac{1}{X}} \emptyset Q Y \\
+\nabla_{Y}^{\perp} \emptyset Q X-\eta(X) \emptyset Q Y-\eta(Y) \emptyset Q X .
\end{gathered}
$$

Using equations (3.4),(3.5)and (2.2) in above equation, we have

$$
\begin{aligned}
& -\eta(X) \emptyset P Y-\eta(Y) \emptyset P X+\emptyset P\left(\nabla_{X} Y\right)+\emptyset Q\left(\nabla_{X} Y\right)+\emptyset P\left(\nabla_{Y} X\right)+\emptyset Q\left(\nabla_{Y} X\right) \\
& +2 B h(X, Y)+2 C h(X, Y)=P\left(\nabla_{X} \varnothing P Y\right)+Q\left(\nabla_{X} \emptyset P Y\right)+\eta\left(\nabla_{X} \varnothing P Y\right) \xi \\
& +P\left(\nabla_{Y} \varnothing P X\right)+Q\left(\nabla_{Y} \varnothing P X\right)+\eta\left(\nabla_{Y} \varnothing P X\right) \xi+h(Y, \varnothing P X)+h(X, \varnothing P Y) \\
& -P A_{\emptyset Q Y} X-Q A_{\varnothing Q Y} X-\eta\left(A_{\emptyset Q Y} X\right) \xi-P A_{\varnothing Q X} Y-Q A_{\emptyset Q X} Y \\
& -\eta\left(A_{\emptyset Q X} Y\right) \xi+\nabla_{X}^{\frac{1}{X}} \emptyset Q Y+\nabla_{Y} \frac{1}{Y} \varnothing Q X .
\end{aligned}
$$

Comparing horizontal, vertical and normal components we get desired result.

\section{Integrability of Distributions}

Theorem4.1. Let $M$ be a semi-invariant submanifold of a nearly hyperbolic Kenmotsu manifold $\bar{M}$ with semisymmetric semi-metric connection. Then the distribution $D \oplus\langle\xi\rangle$ is integrable if the following conditions are satisfied:

for each $X, Y \in(D \oplus\langle\xi\rangle)$.

$$
\begin{aligned}
& S(X, Y) \in(D \oplus\langle\xi\rangle) \\
& h(X, \varnothing Y)=h(\varnothing X, Y)
\end{aligned}
$$

Proof.The torsion tensor $S(X, Y)$ of an almost hyperbolic contact manifold is given by

where $N(X, Y)$ is Neijenhuis tensor. Then

$$
S(X, Y)=N(X, Y)+2 d \eta(X, Y) \xi,
$$

$$
S(X, Y)=[\varnothing X, \varnothing Y]-\varnothing[\varnothing X, Y]-\varnothing[X, \varnothing Y]+2 d \eta(X, Y) \xi .
$$

Suppose that $(D \oplus\langle\xi\rangle)$ is integrable, then $N(X, Y)=0$ for any $X, Y \in(D \oplus\langle\xi\rangle)$.Therefore,

$$
S(X, Y)=2 d \eta(X, Y) \xi \in(D \oplus\langle\xi\rangle) \text {. }
$$


From (4.3), (2.18) and comparing normal part, we have

Replacing $Y$ by $\emptyset Z$, where $Z \in D$, we have

$$
\emptyset Q\left(\nabla_{Y} \varnothing X\right)+C h(Y, \emptyset X)-h(X, Y)=0, \text { for } X, Y \in(D \oplus\langle\xi\rangle) .
$$

Interchanging $X$ and $Z$, we have

$$
\emptyset Q\left(\nabla_{\varnothing Z} \varnothing X\right)+C h(\varnothing Z, \emptyset X)-h(X, \emptyset Z)=0 .
$$

Subtracting (4.4) from (4.5), we obtain

$$
\emptyset Q\left(\nabla_{\emptyset X} \varnothing Z\right)+C h(\varnothing X, \emptyset Z)-h(Z, \varnothing X)=0 .
$$

$$
\varnothing Q[\varnothing X, \varnothing Z]+h(X, \varnothing Z)-h(Z, \varnothing X)=0 .
$$

Since $D \oplus\langle\xi\rangle$ is integrable so that $[\emptyset X, \emptyset Z] \in(D \oplus\langle\xi\rangle)$ for $X, Y \in D$.Consequently above equation gives

$$
h(X, \emptyset Z)=h(\varnothing X, Z) \text {. }
$$

Proposition 4.2. Let $M$ be a semi-invariant submanifold of a nearly hyperbolicKenmotsu manifold $\bar{M}$ with semisymmetric semi-metric connection. Then

for each $Y, Z \in D^{\perp}$.

$$
A_{\varnothing Y} Z-A_{\varnothing Z} Y=\frac{1}{3} \emptyset P[Y, Z]
$$

Proof.Let $Y, Z \in D^{\perp}$ and $X \in T M$. From (3.3), we have

Using (3.1) and (2.9) in (4.6), we have

$$
2 g\left(A_{\emptyset Z} Y, X\right)=g(h(Y, X), \emptyset Z)+g(h(X, Y), \emptyset Z) .
$$

$$
\begin{gathered}
2 g\left(A_{\varnothing Z} Y, X\right)=-g\left(\nabla_{Y} \emptyset X, Z\right)-g\left(\nabla_{X} \emptyset Y, Z\right) \\
-\eta(Y) g(\varnothing X, Z)-\eta(X) g(\varnothing Y, Z) .
\end{gathered}
$$

From (3.2), we have

Replacing $N b y \varnothing Y$

$$
\bar{\nabla}_{X} N=-A_{N} X+\nabla_{X}^{\perp} N-\eta(X) N .
$$

Using (2.5) andabove equation in (4.7), we have

$$
\bar{\nabla}_{X} \emptyset Y=-A_{\emptyset Y} X+\nabla_{X}^{\perp} \emptyset Y-\eta(X) \emptyset Y .
$$

Transvecting $X$ from both sides, we obtain

$$
2 g\left(A_{\emptyset Z} Y, X\right)=-g\left(\varnothing \nabla_{Y} Z, X\right)+g\left(A_{\emptyset Y} Z, X\right) \text {. }
$$

Interchanging $Y$ and $Z$, we have

$$
2 A_{\emptyset Z} Y=-\emptyset \nabla_{Y} Z+A_{\emptyset Y} Z
$$

Subtracting(4.8) from (4.9), we have

$$
2 A_{\emptyset Y} Z=-\emptyset \nabla_{Z} Y+A_{\emptyset Z} Y
$$

$$
\left(A_{\varnothing Y} Z-A_{\varnothing Z} Y\right)=\frac{1}{3} \emptyset P[Y, Z] .
$$

Theorem4.3.Let $M$ be a semi-invariant submanifold of a nearly hyperbolic Kenmotsu manifold $\bar{M}$ with semisymmetric semi-metric connection. Then the distribution $D^{\perp}$ is integrable if and only if

for all $Y, Z \in D^{\perp}$.

$$
A_{\emptyset Y} Z-A_{\emptyset Z} Y=0(4.11)
$$

Proof.Suppose that the distribution $D^{\perp}$ is integrable, that is $[Y, Z] \in D^{\perp}$ for any $Y, Z \in D^{\perp}$, therefore

$$
P[Y, Z]=0 \text {. }
$$

Consequently, from (4.10) we have

$$
A_{\varnothing Y} Z-A_{\emptyset Z} Y=0 \text {. }
$$

Conversely, let (4.11) holds. Then by virtue of (4.10), we have either $P[Y, Z]=0$ or $P[Y, Z]=$ $k \xi$.But $P[Y, Z]=k \xi$ is not possible as $P$ being a projection operator on $D$.So, $P[Y, Z]=0$, this implies that $[Y, Z] \in D^{\perp}$ for all $Y, Z \in D^{\perp}$.

Hence $D^{\perp}$ is integrable.

\section{Parallel Distribution}

Definition 5.1.The horizontal (resp., vertical) distribution $D$ (resp. , $D^{\perp}$ ) is said to be parallel [7] with respect to the connection onMif $\nabla_{X} Y \in D$ (resp. , $\nabla_{Z} W \in D^{\perp}$ ) for any vector field.

Proposition 5.2. Let $M$ be a semi-invariant submanifold of a nearly hyperbolicKenmotsu manifold $\bar{M}$ with semisymmetric semi-metric connection. If the horizontal distribution $D$ is parallel then $h(X, \varnothing Y)=h(Y, \emptyset X)$ for all $X, Y \in D$.

Proof.Let $X, Y \in D$, as $D$ is parallel distribution so that $\nabla_{X} \emptyset Y \in D$ and $\nabla_{Y} \emptyset X \in D$. From (3.19) and (3.20), we have

$$
\begin{aligned}
& Q\left(\nabla_{X} \varnothing P Y\right)+Q\left(\nabla_{Y} \emptyset P X\right)-Q A_{\emptyset Q Y} X-Q A_{\emptyset Q X} Y+h(Y, \emptyset P X)+h(X, \emptyset P Y) \\
& +\nabla_{X}^{\perp} \emptyset Q Y+\nabla_{Y}^{\perp} \emptyset Q X=2 B h(X, Y)+\emptyset Q\left(\nabla_{X} Y\right)+\emptyset Q\left(\nabla_{Y} X\right)+2 C h(X, Y) .
\end{aligned}
$$


As $Q$ being a projection operator on $D^{\perp}$ then we have

$$
h(X, \varnothing Y)+h(Y, \varnothing X)=2 \varnothing h(X, Y) \text {. }
$$

Replacing $X b y \emptyset X$ in (5.1) and using (2.1), we have

$$
h(\varnothing X, \emptyset Y)+h(Y, X)=2 \emptyset h(\varnothing X, Y) \text {. }
$$

Replacing $Y$ by $\varnothing Y$ in (5.1) and using (2.1),we have

Comparing (5.2) and (5.3), we get

$$
h(X, Y)+h(\varnothing Y, \varnothing X)=2 \varnothing h(X, \varnothing Y) .
$$

for all $X, Y \in D$.

$$
h(X, \varnothing Y)=h(Y, \varnothing X)
$$

Definition 5.3.A semi-invariant submanifold is said to be mixed totally geodesic if $h(X, Y)=0$ for all $X \in$ $D$ and $Y \in D^{\perp}$

Proposition 5.4.Let $M$ be a semi-invariant submanifold of a nearly hyperbolicKenmotsu manifold $\bar{M}$ with semisymmetric semi-metric connection. Then $M$ is a mixed totally geodesic if and only if $A_{N} X \in D$ for all $X \in D$.

Proof.Let $A_{N} X \in D$ for all $X \in D$.Now, $g(h(X, Y), N)=g\left(A_{N} X, Y\right)=0$ for $Y \in D^{\perp}$, which is equivalent to $h(X, Y)=0$. Hence $M$ is totally mixed geodesic.

Conversely, Let $M$ is totally mixed geodesic, that is $h(X, Y)=0$ for $X \in D$ and $Y \in D^{\perp}$. Then $g(h(X, Y), N)=$ $g\left(A_{N} X, Y\right)$ gives $g\left(A_{N} X, Y\right)=0$, which implies that $A_{N} X \in D$ for all $Y \in D^{\perp}$.

\section{References}

[1]. Agashe, N. S. and Chafle, M. R., A semi-symmetric non-metric connection in a Riemannian manifold, Indian J. Pure Applied Math., 23 (1992), 399-409.

[2]. Ahmad, M., Semi-invariant submanifolds of nearly Kenmotsu manifold with semi-symmetric semi-metric connection, MathematickiVesnik, 62 (2010), 189-198.

[3]. Ahmad, M.and Ali, K., CR-submanifolds of a nearly hyperbolic Kenmotsu manifold with quarter symmetric non-metric connection, J. Math. Comput. Sci. 3 (2013), No. 3, 905-917.

[4]. --------,Semi-invariant submanifolds of a nearly hyperbolic Kenmotsu manifold with semi-symmetric non-metric connection, IJERA, Vol. 7, No. 4 (2013), 107-119.

[5]. Ahmad, M. and Jun, J. B., On certain class of Riemannian manifold with a semi-symmetric semi-metric connection, Tensor N. S. 72 (2010), 143-151.

[6]. Ahmad M., Jun, J. B. and Siddiqi, M. D., Some properties of semi-invariant submanifolds of a nearly trans-Sasakian manifold admitting a quarter symmetric non-metric connection, JCCMS, 25(1) (2012), 73-90.

[7]. Ahmad, M. and Siddiqi, M. D., On nearly Sasakian manifold with a semi-symmetric semi-metric connection, Int. J. Math. Analysis, 4(35) (2010), 1725-1732.

[8]. - --------, Semi-invariant submanifolds of Kenmotsu manifold immersed in a generalized almost r-contact structure admitting quarter symmetric non-metric connection, Journ. Math.Comput. Sci., 2(4) (2012), 982-998.

[9]. Bejancu, A., CR- submanifolds of a Kaehler manifold. I, Proc. Amer. Math. Soc., 69 (1978), 135-142.

[10]. - -----, Geometry of CR-submanifolds, D. Reidel Publishing Company, Holland, (1986).

[11]. Bejancu, A. and Papaghuic, N., Semi-invariant submanifolds of a Sasakian manifold, An. St. Univ. Al. I. Cuza, Iasi, 27 (1981), 163170 .

[12]. Blair, D. E., Contact manifolds in Riemannian geometry, Lecture Notes in Mathematics, Vol. 509, Springer-Verlag, Berlin, (1976).

[13]. Das, Lovejoy S., Ahmad, M. and Haseeb, A., Semi-invariant submanifolds of a nearly Sasakian manifold endowed with a semisymmetric non-metric connection, Journal of Applied Analysis, 17(1) (2011), 119-130.

[14]. Friedmann, A. and Schouten, J. A., Uber die Geometric der halbsymmetrischenUbertragung Math. Z., 21 (1924), $211-223$.

[15]. Golab, S., On semi-symmetric and quarter symmetric linear connections, Tensor (N.S.), 29(3) (1975), $249-254$.

[16]. Joshi, N. K. and Dube, K. K., Semi-invariant submanifolds of an almost r-contact hyperbolic metric manifold, Demonstratio Math. 36 (2001), 135-143.

[17]. Matsumoto, K., On contact CR-submanifolds of Sasakian manifold, Intern. J. Math. Sci., 6 (1983), 313 -326.

[18]. Shahid, M. H., On semi-invariant submanifolds of a nearly Sasakian manifold, Indian J. Pure and Applied Math., 95(10) (1993), $571-580$.

[19]. Upadhyay, M. D. and Dube, K. K., Almost contact hyperbolic-structure, Acta. Math. Acad. Scient. Hung. Tomus, 28 (1976), 1-4.

[20]. Yano, K. And Kon, M., Contact CR-submanifolds, Kodai Math. J., 5 (1982), 238-252. 